\title{
Isolation and characterization of Uropathogenic Escherichia coli (UPEC) from red panda (Ailurus fulgens)
}

Songrui Liu' ${ }^{1,2,3+}$, Yunli Li $i^{1,2,3 \dagger}$, Chanjuan Yue ${ }^{1,2,3}$, Dongsheng Zhang ${ }^{1,2,3}$, Xiaoyan Su ${ }^{1,2,3}$, Xia Yan ${ }^{1,2,3}$, Kuixing Yang ${ }^{1,2,3}$, Xin Chen ${ }^{1,2,3}$, Guifu Zhuo ${ }^{1,2,3}$, Tong Cai ${ }^{4,5}$, Jiangfeng Liu ${ }^{4,5}, X_{\text {P Peng }}^{4,5^{*}}$ and Rong Hou ${ }^{1,2,3^{*}}$

\begin{abstract}
Background: Disease prevention and control is a significant part in the ex-situ conservation of the endangered red panda (Ailurus fulgens), being bacterial infection is one of the most important health threats to the captive population. To date, studies about the infection caused by Escherichia coli in the red panda are scarce. This study was conducted to determine the cause of death of a captive red panda through clinical symptoms, complete blood count, biochemical analysis, pathological diagnosis and bacterial whole genome sequencing.

Case presentation: The following report describes a case of a 1.5 year old captive red panda (Ailurus fulgens) that was found lethargic and anorectic. She was moved to the quarantine area for daily treatment with $50 \mathrm{mg}$ of Cefpodoxime Proxetil. During the three-day treatment, she did not eat or defecate, and then died. Clinical hematology revealed the values of neutrophils, alanine aminotransferase (ALT), aspartate aminotransferase (AST) and blood urea nitrogen (BUN) were significantly higher. Histological analysis demonstrated major pathological damage in the kidneys, liver and lungs, characterized by hyperemia, parenchymal cell degeneration and necrosis and inflammatory cell infiltration which were predominantly neutrophilic. A bacterial strain confirmed as Escherichia coli was isolated post mortem. Whole genome sequencing of the E. coli showed the complete genome size was 4.99 Mbp. PapA, PapC, OmpA, OmpU and other virulence factors which specific to Uropathogenic Escherichia coli (UPEC) were found in the isolate. Among the virulence factors, P pili, type I pili and related factors of the iron uptake system were associated with nephrotoxicity.
\end{abstract}

Conclusion: The red panda died of bacterial infection caused by an uropathogenic strain of Escherichia coli. The pathogenic mechanisms of the strain are closely related to the expression of specific virulence genes.

Keywords: Red panda, Uropathogenic Escherichia coli, Pathological diagnosis

\footnotetext{
* Correspondence: pengxi197313@163.com; 405536517@qq.com

${ }^{\dagger}$ Songrui Liu and Yunli Li contributed equally to this work.

${ }^{4}$ Key Laboratory of Southwest China Wildlife Resources Conservation

(Ministry of Education), Nanchong 637009, Sichuan, China

${ }^{1}$ Chengdu Research Base of Giant Panda Breeding, Chengdu 610081,

Sichuan, China

Full list of author information is available at the end of the article
}

(c) The Author(s). 2020 Open Access This article is licensed under a Creative Commons Attribution 4.0 International License, which permits use, sharing, adaptation, distribution and reproduction in any medium or format, as long as you give appropriate credit to the original author(s) and the source, provide a link to the Creative Commons licence, and indicate if changes were made. The images or other third party material in this article are included in the article's Creative Commons licence, unless indicated otherwise in a credit line to the material. If material is not included in the article's Creative Commons licence and your intended use is not permitted by statutory regulation or exceeds the permitted use, you will need to obtain permission directly from the copyright holder. To view a copy of this licence, visit http://creativecommons.org/licenses/by/4.0/ The Creative Commons Public Domain Dedication waiver (http://creativecommons.org/publicdomain/zero/1.0/) applies to the data made available in this article, unless otherwise stated in a credit line to the data. 


\section{Background}

Escherichia coli (E. coli) is a common species of bacteria distributed in the digestive tract of humans and animals, but most of the strains have no pathogenic affect. Some strains may acquire virulence factors, turning into pathogenic bacteria, causing infections in animals and humans [1]. E. coli infection occurs in poultry and livestock, as well as in wild animals such as snub-nosed monkeys and giant pandas [2-5]. Uropathogenic Escherichia coli (UPEC) can induce urinary tract infection through entering the urethra, then causing pyelonephritis, cystitis and urethritis [6-8]. If the bacterial infections cannot be controlled in time, renal function damage may occur and pose a serious threat to health and life.

The red panda (Ailurus fulgens) has been classified as an endangered species by the International Union for the Conservation of Nature (IUCN) as its wild population is estimated at less than 10,000 mature individuals, and its survival in the wild is threatened by deforestation, loss of habitat and fragmentation of the existing wild populations [9]. Disease prevention and control is a significant part in the ex-situ conservation of the red panda, being bacterial infection is one of the most important health threats to the captive population. To date, studies about the infection caused by Escherichia coli in the red panda are scarce. Analysis of post-mortem reports is an important tool in increasing our understanding of the red panda in captivity and improving our husbandry and management procedures for this species [10]. Due to its special ecological status, prevention and treatment of $E$. coli infection in the red panda needs high attention.

This study was conducted to determine the cause of death, etiology and pathogenesis in a red panda. The cause of death was identified through clinical symptoms, complete blood count, biochemical analysis, gross and histopathological diagnosis. A strain of E. coli was isolated and identified, and bacterial whole genome sequencing was used to explore the pathogenic mechanism of the bacteria. The results provide a scientific basis for the diagnosis and clinical treatments of the bacterial infection in the red panda.

\section{Case presentation}

\section{Clinical history}

A 1.5 year old captive female red panda (Ailurus fulgens) was found to be lethargic and anorectic at Panda Valley, the Chengdu Field Research Center for Giant Pandas in Dujiangyan, China. Oral and nasal secretions were collected for the search of Canine Distemper Virus (CDV) and Canine Parvovirus (CPV) using AsanEsay Test (ASAN PHARM. Co., LTD), and the results were negative. It was anaesthetized with $21 \mathrm{mg}$ Zoletil (intramuscular injection) at a dose of $3 \mathrm{mg} / \mathrm{kg}$ body weight, and treated with an intravenous injection of $350 \mathrm{mg}$ Sulperazon (Cefoperazone Sodium and Sulbactam Sodium) in $150 \mathrm{~mL} 0.9 \% \mathrm{NaCl}$. The complete blood count and biochemical analysis results are shown in Additional file 1. Compared with a previous report on blood counts and biochemical parameters of 28 healthy red pandas, the values of neutrophils, alanine aminotransferase (ALT), aspartate aminotransferase (AST) and blood urea nitrogen (BUN) found in this study were significantly higher while remaining parameters were normal [11]. She was moved to the quarantine area of the Chengdu Research Base of Giant Panda Breeding for daily treatment of oral Cefpodoxime Proxetil $50 \mathrm{mg}$. During the three-day treatment, she did not eat or defecate, and then died.

\section{Necropsy}

During necropsy, the red panda's skin and fur was intact, and no traumatic injuries were observed. Both kidneys were swollen with several white pinpoint spots on the cutting surface, with white cord-like streaks in the medulla. The liver was icteric, with soft and brittle texture, and few dark red ecchymoses on the cutting surface. The lungs were dark red with multifocal plaques of black-red hemorrhage. The spleen was swollen and dark red with a small number of black-red plaques. The heart and intestines were intact, with no obvious lesions.

\section{Histopathology}

Specimens, including the heart, liver, spleen, lung, kidney, gastrointestinal tract and other tissues were fixed in $4 \%$ paraformaldehyde, and routinely processed in paraffin. Sections $(5 \mu \mathrm{m})$ were stained with haematoxylin and eosin $\mathrm{Y}(\mathrm{H} \& \mathrm{E})$ and evaluated for histopathological changes under the microscope (Leica DM4B optics). Histopathological analysis revealed significant glomerular congestion in the renal cortex, necrosis of renal tubular epithelial cells, collapse of renal tubular lumens and interstitial infiltration of neutrophils, lymphocytes, plasma cells and macrophages (Fig. 1a). Cord-like interstitial inflammatory lesions were noted in the renal medulla regions, as normal collecting duct structures were replaced by extensive inflammation (Fig. 1b). In the liver, there was vesicular degeneration or steatosis of hepatocytes, mild congestion, and perivascular inflammatory cell (neutrophils, macrophages and lymphocytes) infiltration (Fig. 1c). In the lung, alveolar walls were thickened by lymphoplasmacytic infiltration and congestion. Some alveolar spaces collapsed, while others were dilated due to compensatory emphysema (Fig. 1d). Expanded lymphoid follicular germinal centers showed increased splenic white pulp, while red pulp showed congestion, hemorrhage and inflammation with nuclear debris (Fig. 1e). Mesenteric lymph node sinuses were dilated with increased macrophages and lymphocytes (Fig. 1f). 

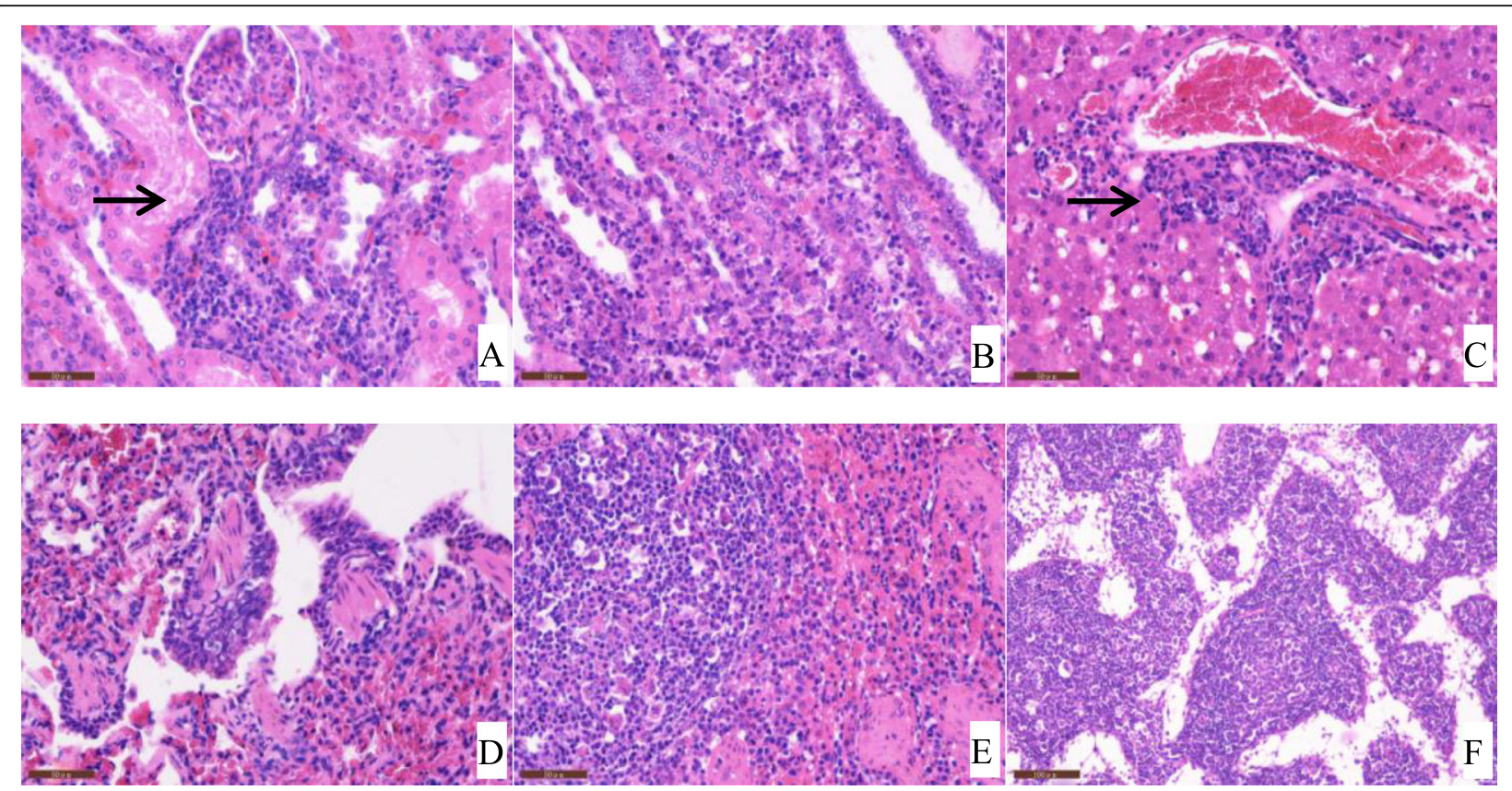

Fig. 1 Histopathological findings with UPEC infection. a: Glomerular congestion, epithelial cell necrosis in small renal tubules, and focal infiltration of inflammatory neutrophils and fewer lymphocytes (arrow). b: Cord-like infiltration of lymphocytes and plasma cells in the renal medullary interstitium. c: Mild hepatic congestion, perivascular infiltration of lymphocytes and plasma cells (arrow), and hepatocyte vacuolar degeneration. d: Thickening, hyperemia of the pulmonary alveolar wall and infiltration of lymphocytes and plasma cells. e: Congestion and hemorrhage in splenic red pulp. $\mathbf{f}$ : Expansion of the mesenteric lymph node sinuses by infiltration of lymphocytes and plasma cells. H\&E, bar $=50 \mu \mathrm{m}$

Other tissues (heart, pancreas, stomach, digestive tract) did not show any pathological lesion.

\section{Bacterial isolation and molecular identification}

Strains were isolated from the kidney, liver and lung samples. Genomic DNA was individually extracted from the isolates using the TIANamp Bacteria DNA kit according to the manufacturer's instructions (Tiangen Biotech Co., Ltd., Beijing, China). The 16S rRNA gene was amplified by PCR performed in a T100 ${ }^{\mathrm{ma}}$ Thermal Cycler (Bio-rad, USA). The amplified 1500 bp 16S rRNA genes of the isolated strains shared the highest identity (99.45\%) with that of E. coli (GenBank: LC050175.1), and the phylogenetic analysis demonstrated that the isolated strain and $E$. coli strains have high phylogenetic relatedness using the Neighbor-Joining method (Fig. 2). The sequence of the isolated strain was submitted to GenBank under the accession number MT820501.

\section{Whole genome sequencing}

Genomic DNA was extracted using a sample of highquality, high-molecular-weight DNA prepared using the TIANamp Bacteria DNA kit according to the manufacturer's instructions (Tiangen Biotech Co., Ltd., Beijing, China). The sample was sent to an external laboratory for whole genome sequencing using the PacBio RS II platform (completed by Biomarker Technologies Co, LTD.). The complete genome size of the E. coli chromosome isolated from the red panda was 4,990,420 bp (GenBank: PRJNA668015). BLAST alignment of the predicted protein sequence with the Antibiotic Resistance Gene Database (ARDB) showed that the strain contained 20 resistance genes (Additional file 2) including acra, acrb, mdte, mdtf, mdtn, mdto, mdtp, tolc, arna, baca, bcr, bl1_ec, emre, ksga, macb, mdfa, mdtg, mdth, mdtk and mdtl. These genes can mediate resistance to aminoglycosides, $\beta$-lactams, macrolides and other antibiotics through resistance-nodulationcell division transporter system, multidrug resistance efflux pump and other ways. BLAST alignment with the Virulence Factor Database (VFDB) showed that the measured E. coli contained 713 virulence factors (Additional file 3), including outer membrane protein, flagella, P pili, S pili, type I pili, cytotoxic necrosis factor, and hemolysin. In addition, factors related to the iron uptake system and other types of systems were identified. Among these virulence factors, PapA, PapC, OmpA, OmpU and other virulence factors were specific to Uropathogenic Escherichia coli (UPEC) [8], thus confirming its identity.

\section{Discussion and conclusions}

Understanding and addressing disease threats in the exsitu population of the red panda is crucial to the conservation of in-situ populations of this species in China. In this study, blood analysis revealed leukocytosis with 


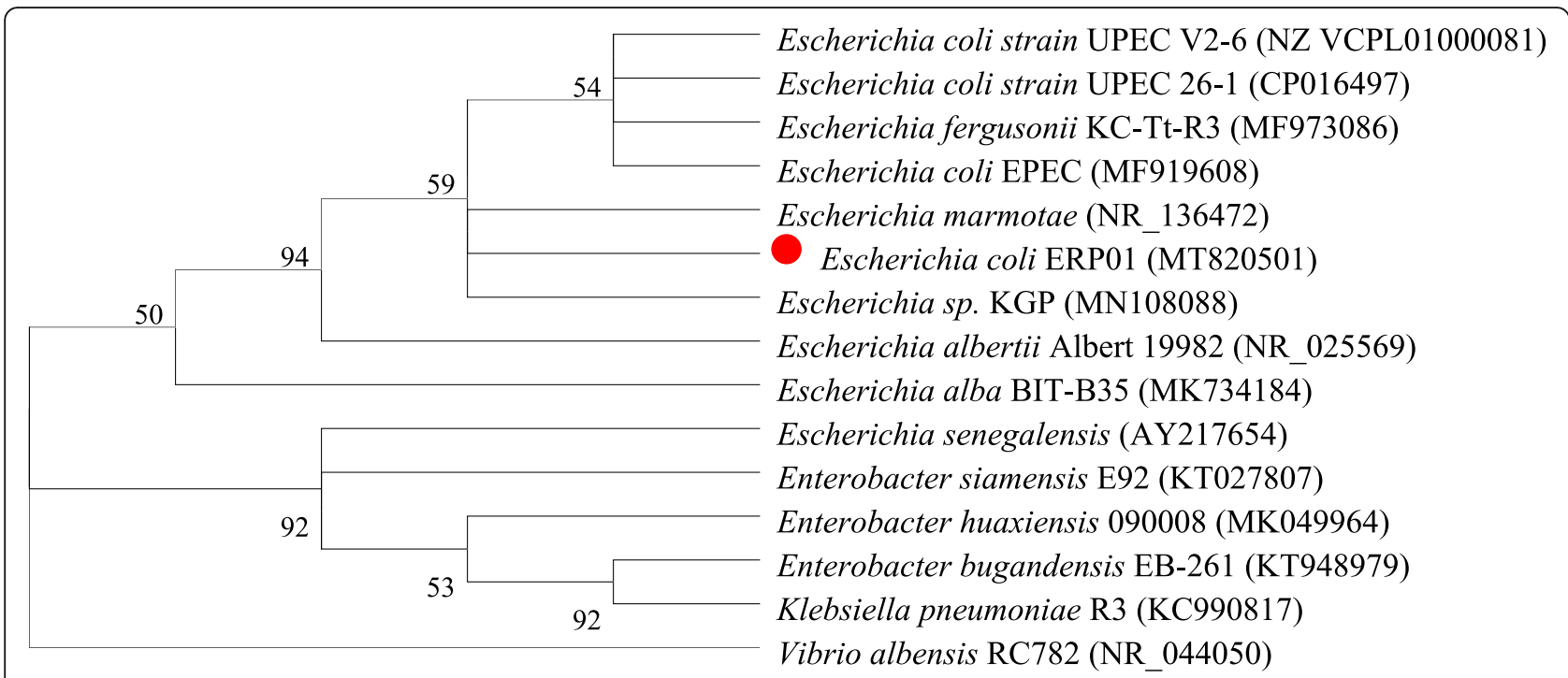

Fig. 2 Phylogenetic analysis of the isolated strain and close related species using the Neighbor-Joining method. Bootstrap values (expressed as a percentage of 1000 replications) $>50 \%$ are given at the branching points. Vibrio albensis strain RC782 (NR_044050) was used as outgroup

increased ALT, AST and BUN, suggesting that bacterial infection caused liver and kidney damage, followed by death [12-14]. Histopathological findings revealed major pathological damage in the kidneys, liver and lungs, characterized by hyperemia, parenchymal cell degeneration and necrosis, inflammatory cell infiltration which was predominantly neutrophilic. These findings are consistent with the results of the complete blood count and biochemical analysis. Researchers have reported acute pyelonephritis caused by UPEC in the mouse model, with renal histopathological lesions including infiltration of inflammatory cells (predominantly neutrophils) in renal pelvis submucosa, renal interstitial dilatation, partial renal tubular epithelial cell necrosis and shedding [15-17]. These were similar to the pathological manifestations of the kidney in this red panda. We conclude that UPEC induced pyelonephritis in this red panda, through retrograde infection from the urethra, then lead to septicemia.

Although copy numbers of acra、tolc、 and emre genes (included in multi-drug efflux systems which mediate the aminoglycoside, polymyxin and beta_lactam antibiotic resistance) were detected, this $E$. coli isolate was not sensitive to Gentamycin and Cefoperazone. As far as the clinical treatment of the red panda, when symptoms were first noted, Sulperazon (Cefoperazone Sodium and Sulbactam Sodium) and Gentamicin were used, but there was no obvious curative effect. Current increasing usage of the clinical antibacterial drugs forces antibiotic resistance to occur in both pathogenic and zoonotic bacteria in animals [18]. Previous research showed that the antibiotic resistance strains can make treatment of bacterial infections more difficult, leading an overall increase in transmission, morbidity and mortality $[19,20]$. These results suggest that effective treatment should be based on the results of antimicrobial susceptibility tests and clinical efficacy in the control of bacterial infections in clinical practice.

Previous researchers found that $80 \%$ of E. coli containing P pili may cause pyelonephritis [21]. In our study, the whole genome sequencing results found high express of $\mathrm{P}$ pili and typeIpili genes. The P pili receptors exist on renal epithelia, while type I pili mediates the biofilm formation and colonization of the bacteria in the renal epithelia [8], which makes the strain more nephrotoxic. In addition, the virulence factors associated with the iron uptake system are also high in this case. The presence of the iron uptake system enhances the pathogenicity of the bacteria, contributing to the bacterial survival through the host's heme and ferritin [22].

Based on the results obtained, we can conclude the red panda died of septicemic bacterial infection and the isolated bacterial strain was identified as UPEC. Since this is the first report of UPEC strain isolation in the red panda, further research is needed for a better understanding of the epidemiology, susceptibility and antibiotic resistance of these types of $E$. coli strains in the red panda.

\section{Supplementary Information}

Supplementary information accompanies this paper at https://doi.org/10. 1186/s12917-020-02624-9.

Additional file 1. Complete blood count and biochemical analysis for the red panda.

Additional file 2. Antibiotic resistance protein of the $E$. coli chromosome isolated from the red panda.

Additional file 3. Virulence factor protein of the E. coli chromosome isolated from the red panda. 


\section{Abbreviations}

UPEC: Uropathogenic Escherichia coli; IUCN: International Union for Conservation of Nature; CBC: Complete blood count; ARDB: Antibiotic resistance gene database; VFDB: Virulence factor database; CDV: Canine distemper virus; CPV: Canine parvovirus; WBC: White blood cells; NEU: Neutrophils; RBC: Red blood cells; HGB: Hemoglobin; MCH: Mean corpuscular hemoglobin; MCHC: Mean corpuscular hemoglobin concentration; PLT: Platelets; TP: Total protein; TB: Total bilirubin; ALT: Alanine aminotransferase; AST: Aspartate aminotransferase; CK: Creatine kinase; TC: Total cholesterol; BUN: Blood urea nitrogen; CR: Creatinine; UA: Uric acid; GLU: Glucose; BHI: Brain heart infusion; IACUC: Institutional Animal Care and Use Committee

\section{Acknowledgements}

The authors expressed their thanks to the veterinary staff of the Chengdu Research Base of Giant Panda Breeding for their participation in necropsy work of this study. In addition, we would like to thank James E Ayala and Rita McManamon for their suggestions and help in this study.

\section{Authors' contributions}

$S L$ and $Y L$ designed the work and performed main experimental operation, and were major contributors in writing the manuscript; $C Y, D Z, X S, X Y, K Y$, $X C$ and GZ performed the necropsy work and acquired the samples.TC and $J \mathrm{~L}$ participated the experimental operation; $\mathrm{RH}$ and XP verified all the data, figures and materials (including reagents), and proofreading. All authors read and approved the final manuscript.

\section{Funding}

This work was funded by the Basic Research Project of the Science and Technology Department of Sichuan Province (2017JY0099). The funders had no role in the design of experiments, data collection and analysis, decision to publish, or preparation of the manuscript.

\section{Availability of data and materials}

All the nucleotide sequences are deposited in GenBank and available under accession numbers MT820501 and PRJNA668015. Other data generated or analyzed during this study are included in additional files.

\section{Ethics approval and consent to participate}

The use of materials and all experimental procedures involving animals were approved by Institutional Animal Care and Use Committee of the Chengdu Research Base of Giant Panda Breeding protocol \#2019013.

\section{Consent for publication}

Not applicable.

\section{Competing interests}

We are sure that there is no potential conflict of interest and no part of this paper has published or submitted anywhere

\section{Author details}

${ }^{1}$ Chengdu Research Base of Giant Panda Breeding, Chengdu 610081, Sichuan, China. ${ }^{2}$ Sichuan Key Laboratory of Conservation Biology for Endangered Wildlife, Chengdu 610081, Sichuan, China. ${ }^{3}$ Sichuan Academy of Giant Panda, Chengdu 610081, Sichuan, China. ${ }^{4}$ Key Laboratory of Southwest China Wildlife Resources Conservation (Ministry of Education), Nanchong 637009, Sichuan, China. ${ }^{5}$ College of Life Science, China West Normal University, Nanchong 637009, Sichuan, China.

Received: 9 December 2019 Accepted: 15 October 2020 Published online: 27 October 2020

\section{References}

1. Pupo GM. Evolutionary relationships among pathogenic and nonpathogenic Escherichia coli strains inferred from multilocus enzyme electrophoresis and mdh sequence studies. Infect Immun. 1997:65:2685.

2. Guo D-Z, Hu X-Y, Zhou S-Q, Li J-K, Wang M-Q, Du Y-S. Pathological observation of septicemia on a Golden monkey infected by Klebsiell pneumoniae and Escherichia coli. Huazhong Agric University. 2001;20:60-2.
3. Lutful Kabir SM. Avian colibacillosis and salmonellosis: a closer look at epidemiology, pathogenesis, diagnosis, control and public health concerns. Int J Env Res Pub Heal. 2010;7:89-114.

4. Guo L, Long M, Huang Y, Wu G, Deng W, Yang X. Antimicrobial and disinfectant resistance of Escherichia coli isolated from giant pandas. Appl Microbiol. 2015;119:55-64.

5. Luppi A. Swine enteric colibacillosis: diagnosis, therapy and antimicrobial resistance. Porcine Heal Manag. 2017;3:16-34.

6. Van Hoek AH, Stalenhoef JE, Duijkeren EV, Franz E. Comparative virulotyping of extended-spectrum cephalosporin-resistant E. coli isolated from broilers, humans on broiler farms and in the general population and UTI patients. Vet Microbiol. 2016;194:55-61.

7. Freitag T, Squires RA, Schmid J, Elliott J. Feline uropathogenic Escherichia coli from Great Britain and New Zealand have dissimilar virulence factor genotypes. Vet Microbiol. 2005;106:79-86.

8. Bien J, Sokolova O, Bozko P. Role of Uropathogenic Escherichia coli virulence factors in development of urinary tract infection and kidney damage. Int J Nephrol. 2012;2012:681473.

9. Wei F-W, Feng Z-J, Wang Z-W, Hu J-C. Current distribution, status and conservation of wild red pandas Ailurus fulgens in China. Biol Conserv. 1999; 89:285-91.

10. Brian P. Red Panda Pathology. In: Angela RG, editor. Red Panda: Biology and Conservation of the First Panda. London: Academic Press; 2011. p. 287-302.

11. Xu S-H, Xiu Y-F, Shao L-P, Wang D-C, Chen T, Shi F-N, Chen Y-C. Determination of blood physiology and biochemistry parameters of Ailurus fulgens. J Econ Anim. 2009:13:1-3.

12. Liu C-H, Lehan C, Speer ME, Fernback DJ, Rudolph AJ. Dgenerative changes in neutrophils-an indicator of bacterial infection. Pediatr Res. 1984;18:280A.

13. Moreno BA, González ML, Mendoza-Jiménez J, García-Buey L, Moreno OR. Utility of analytical parameters in the diagnosis of liver disease. An Med Interna. 2007;24:38.

14. Shavit L, Lifschitz M, Galperin I. Influence of enteric nutrition on blood urea nitrogen (BUN) in very old patients with chronic kidney disease (CKD). Arch Gerontol Geriat. 2012;54:228-31.

15. Lane MC, Mobley HLT. Role of P-fimbrial-mediated adherence in pyelonephritis and persistence of Uropathogenic Escherichia coli (UPEC) in the mammalian kidney. Kidney Int. 2007:72:19-25.

16. Wei Y, Li K, Wang N, Cai G-D, Zhang T, Lin Y. Activation of endogenous anti-inflammatory mediator cyclic amp attenuates acute pyelonephritis in mice induced by Uropathogenic Escherichia coli. Am J Pathol. 2015;185:47284.

17. Li K, Wu K-Y, Wu W, Wang N, Zhang T, Choudhry N. C5ar1 promotes acute pyelonephritis induced by Uropathogenic E. coli. JCI Insight. 2017;2:e97626.

18. Delsol AA, Randall L, Cooles S, Woodward MJ, Sunderland J, Roe JM. Effect of the growth promoter avilamycin on emergence and persistence of antimicrobial resistance in enteric bacteria in the pig. Appl Microbiol. 2005: 98:564-71.

19. Rocha P, Baleeiro C, Tunkel AR. Impact of antimicrobial resistance on the treatment of invasive pneumococcal infections. Curr Infect Dis Rep. 2000;2: 399-408.

20. Abatih EN, Alban L, Ersbø\|l AK, MD, Wong LF. Impact of antimicrobial usage on the transmission dynamics of antimicrobial resistant bacteria among pigs. J Theor Biol. 2009;256:561-73.

21. Dufour N, DebarbieuxL MF, Ricard JD. Treatment of highly virulent extraintestinal pathogenic Escherichia coli pneumonia with bacteriophages. Crit Care Med. 2015;43:e190.

22. Braun $\mathrm{V}$. Iron uptake mechanisms and their regulation in pathogenic bacteria. Int J Med Microbiol. 2001;291:67-79.

\section{Publisher's Note}

Springer Nature remains neutral with regard to jurisdictional claims in published maps and institutional affiliations. 